VOLUME 25 (2019) 212-223

DOI: $10.24330 /$ ieja.504155

\title{
ON 2-ABSORBING MODULES OVER NONCOMMUTATIVE RINGS
}

\author{
N. J. Groenewald and Bac T. Nguyen \\ Received: 27 July 2018; Revised: 27 October 2018; Accepted: 31 October 2018 \\ Communicated by Sait Halıcıŏlu
}

\begin{abstract}
Let $R$ be a noncommutative ring with identity. We define the notion of a 2-absorbing submodule and show that if the ring is commutative then the notion is the same as the original definition of that of A. Darani and

F. Soheilnia. We give an example to show that in general these two notions are different. Many properties of 2-absorbing submodules are proved which are similar to the results for commutative rings.
\end{abstract}

Mathematics Subject Classification (2010): 16N60

Keywords: 2-Absorbing submodule, strong 2-absorbing submodule, prime submodule, completely prime submodule

\section{Introduction}

In 2007, Badawi [1] introduced the concept of 2-absorbing ideals of commutative rings with identity, which is a generalization of prime ideals, and investigated some properties. He defined a 2-absorbing ideal $P$ of a commutative ring $R$ with identity to be a proper ideal of $R$ and if whenever $a, b, c \in R$ with $a b c \in P$, then $a b \in P$ or $b c \in P$ or $a c \in P$. In 2011, Darani and Soheilnia [3] introduced the concept of 2-absorbing submodules of modules over commutative rings with identities. A proper submodule $P$ of a module $M$ over a commutative ring $R$ with identity is said to be a 2-absorbing submodule of $M$ if whenever $a, b \in R$ and $m \in M$ with $a b m \in P$, then $a b M \subseteq P$ or $a m \in P$ or $b m \in P$. One can see that 2-absorbing submodules are generalization of prime submodules. Moreover, it is obvious that 2-absorbing ideals are special cases of 2-absorbing submodules.

Throughout all rings (not necessarily commutative rings) have identities and all modules are unital left modules. In recent years the study of the absorbing property of rings, modules and related notions have been some of the topics of interest in the development of the ring and module theory. In this paper we study the notion of 2-absorbing modules over noncommutative rings. We prove basic properties of 2-absorbing submodules analogous to properties studied by Payrovi and Babaei in 
[6] and [7] for 2-absorbing submodules over commutative rings. We also introduce the notion of strong 2-absorbing submodules and show that in general if $R$ is not a commutative ring then the notions of 2-absorbing and strong 2-absorbing submodules are not the same. If $R$ is a commutative ring then the notions of 2-absorbing and strong 2-absorbing submodule coincide with that of the original definition introduced by Darani and Soheilnia in [3].

\section{2-Absorbing submodules and ideals}

Prime submodules of modules over associative rings were introduced by Dauns [4]. A proper submodule $N$ of $M$ is called prime, if $r R m \subseteq N$ implies $r M \subseteq N$ or $m \in N$ for all $r \in R$ and $m \in M$. Recently the concept of 2-absorbing submodules was introduced by Darani and Soheilnia as a generalization of 2-absorbing ideals in $[3]$.

Definition 2.1. Let $P$ be a proper ideal of a ring $R$. Then $P$ is a 2-absorbing ideal of $R$ if $a R b R c \subseteq P$ implies $a b \in P$ or $b c \in P$ or $a c \in P$ for all $a, b, c \in R$.

Definition 2.2. Let $R$ be a ring and $N$ be a proper submodule of an $R$-module $M$. Then $N$ is 2-absorbing submodule of $M$ if $a R b R m \subseteq N$ implies $a b M \subseteq N$ or $a m \in N$ or $b m \in N$ for all $a, b \in R$ and $m \in M$.

Remark 2.3. If $R$ is a commutative ring then this notion of a 2-absorbing submodule coincides with that of Darani and Soheilnia.

Definition 2.4. Let $R$ be a noncommutative ring and $M$ an $R$-module. We define a proper submodule $P$ of $M$ to be a strong 2-absorbing submodule if whenever $a, b \in R$ and $m \in M$ with $a b m \in P$, then $a b M \subseteq P$ or $a m \in P$ or $b m \in P$.

Proposition 2.5. Let $R$ be a ring and $N$ be a prime submodule of an $R$-module $M$. If $a R b R m \subseteq N$ and am $\notin N$, then $b M \subseteq N$ for all $a, b \in R$ and $m \in M$.

Proof. Let $a, b \in R$ and $m \in M$. Assume that $a R b R m \subseteq N$ and $a m \notin N$. First, we show that $b R m \subseteq N$. Let $r$ be any element of the ring $R$. Then $a R(b r m) \subseteq$ $a R(b R m) \subseteq N$. Since $N$ is a prime submodule, $a M \subseteq N$ or brm $\in N$. Then $b r m \in N$ because $a m \notin N$. That is $b R m \subseteq N$. Since $N$ is a prime submodule and am $\notin N$, it follows that $m \notin N$ so that $b M \subseteq N$.

From [5], a proper submodule $P$ of an $R$-module $M$ is called completely prime, if $a m \in P$ implies $m \in P$ or $a M \subseteq P$, for each $a \in R$ and $m \in M$. An $R$-module $M$ is completely prime if the zero submodule of $M$ is a completely prime submodule 
of $M$. In general, an $R$-module $M / P$ is a completely prime module if and only if $P$ is a completely prime submodule of $M$.

Proposition 2.6. If $N$ is a prime (completely prime) submodule of an $R$-module $M$, then $N$ is a 2-absorbing (strong 2-absorbing) submodule of $M$.

Proof. Assume that $N$ is a prime (completely prime) submodule of an $R$-module $M$. Let $a, b \in R, m \in M$ and assume $N$ is a prime submodule such that $a R b R m \subseteq$ $N$ but $a m \notin N$. Thus $b M \subseteq N$ by Proposition 2.5. Then $b m \in N$ and $a b M \subseteq a N \subseteq$ $N$. Hence $N$ is a 2-absorbing submodule of $M$. Now, assume $N$ is a completely prime submodule and that $a b m \in N$ but $b m \notin N$. Since $N$ is completely prime and $b m \notin N$, we have $a M \subseteq N$. Hence $a b M \subseteq a M \subseteq N$ and we have $N$ is a strong 2-absorbing submodule of $M$.

In general if $R$ is not a commutative ring then the notions of 2 -absorbing and strong 2-absorbing submodules are not the same.

Example 2.7. Let $M=\left\{\left(\begin{array}{cc}\overline{0} & \overline{0} \\ \overline{0} & \overline{0}\end{array}\right),\left(\begin{array}{cc}\overline{1} & \overline{1} \\ \overline{0} & \overline{0}\end{array}\right),\left(\begin{array}{cc}\overline{0} & \overline{0} \\ \overline{1} & \overline{1}\end{array}\right),\left(\begin{array}{cc}\overline{1} & \overline{1} \\ \overline{1} & \overline{1}\end{array}\right)\right\}$ where entries of matrices in $M$ are from $\mathbb{Z}_{2}=\{\overline{0}, \overline{1}\}$ and $R=M_{2}(\mathbb{Z}) .{ }_{R} M$ is a simple module and hence also a prime module and as such 2-absorbing. However, $M$ is not a strong 2-absorbing module.

Proof. It is clear that ${ }_{R} M$ is a simple module and hence also a prime module.

$$
\begin{gathered}
\text { Take } a=\left(\begin{array}{ll}
2 & 1 \\
2 & 4
\end{array}\right) \text { and } b=\left(\begin{array}{ll}
1 & 0 \\
3 & 5
\end{array}\right) \in R \text { and } m=\left(\begin{array}{ll}
\overline{1} & \overline{1} \\
\overline{1} & \overline{1}
\end{array}\right) \in M . \\
a b m=\left(\begin{array}{ll}
2 & 1 \\
2 & 4
\end{array}\right)\left(\begin{array}{ll}
1 & 0 \\
3 & 5
\end{array}\right)\left(\begin{array}{ll}
\overline{1} & \overline{1} \\
\overline{1} & \overline{1}
\end{array}\right)=\left(\begin{array}{ll}
\overline{0} & \overline{0} \\
\overline{0} & \overline{0}
\end{array}\right) \text { but } \\
a m=\left(\begin{array}{ll}
2 & 1 \\
2 & 4
\end{array}\right)\left(\begin{array}{ll}
\overline{1} & \overline{1} \\
\overline{1} & \overline{1}
\end{array}\right)=\left(\begin{array}{ll}
\overline{1} & \overline{1} \\
\overline{0} & \overline{0}
\end{array}\right) \neq\left(\begin{array}{ll}
\overline{0} & \overline{0} \\
\overline{0} & \overline{0}
\end{array}\right), \\
b m=\left(\begin{array}{ll}
1 & 0 \\
3 & 5
\end{array}\right)\left(\begin{array}{ll}
\overline{1} & \overline{1} \\
\overline{1} & \overline{1}
\end{array}\right)=\left(\begin{array}{ll}
\overline{1} & \overline{1} \\
\overline{0} & \overline{0}
\end{array}\right) \neq\left(\begin{array}{ll}
\overline{0} & \overline{0} \\
\overline{0} & \overline{0}
\end{array}\right) \\
\text { and } a b M \neq\left\{\left(\begin{array}{ll}
\overline{0} & \overline{0} \\
\overline{0} & \overline{0}
\end{array}\right)\right\} \text { since } \\
a b\left(\begin{array}{ll}
\overline{1} & \overline{1} \\
\overline{0} & \overline{0}
\end{array}\right)=\left(\begin{array}{ll}
2 & 1 \\
2 & 4
\end{array}\right)\left(\begin{array}{ll}
1 & 0 \\
3 & 5
\end{array}\right)\left(\begin{array}{ll}
\overline{1} & \overline{1} \\
\overline{0} & \overline{0}
\end{array}\right)=\left(\begin{array}{ll}
\overline{1} & \overline{1} \\
\overline{0} & \overline{0}
\end{array}\right) \neq\left(\begin{array}{ll}
\overline{0} & \overline{0} \\
\overline{0} & \overline{0}
\end{array}\right) .
\end{gathered}
$$

In [2] a module is defined to be a semi-commutative module if whenever $a m=0$ for $a \in R$ and $m \in M$, we have $a R m=0$. A submodule $N$ of an $R$-module $M$ is a semi-commutative submodule if whenever $a m \in N$ for $a \in R$ and $m \in M$, we have $a R m \subseteq N$. 
Proposition 2.8. Let $M$ be a left $R$ module. If $N$ is a 2-absorbing submodule which is also a semi-commutative submodule, then $N$ is a strong 2-absorbing submodule.

Proof. Suppose $N$ is 2-absorbing. Let $a, b \in R$ and $m \in M$ such that $a b m \in N$. Since $N$ is a semi-commutative submodule, we have $a R b R m \subseteq N$. Now $N 2$ absorbing implies that $a m \in N$ or $b m \in N$ or $a b M \subseteq N$. Hence $N$ is a strong 2-absorbing submodule of $M$.

Compare the next Theorem with Theorem 2.3 in [6].

Theorem 2.9. Let $N$ be a proper submodule of an $R$-module $M$. If $N$ is a 2absorbing submodule of $M$, then $\left(N:_{R} M\right)=\{r \in R: r M \subseteq N\}$ is a 2-absorbing ideal of $R$.

Proof. Let $a, b, c \in R$ such that $a R b R c \subseteq\left(N:_{R} M\right)$ and suppose $a c \notin\left(N:_{R} M\right)$ and $b c \notin\left(N:_{R} M\right)$. We show that $a b \in\left(N:_{R} M\right)$. Since $a c, b c \notin\left(N:_{R} M\right)$, there exists $x_{1}, x_{2} \in M$ such that $a c x_{1} \notin N$ and $b c x_{2} \notin N$. Now, $a R b R c\left(x_{1}+x_{2}\right) \subseteq N$. Since $N$ is a 2-absorbing submodule of $M$ we have $a b \in\left(N:_{R} M\right)$ or $a c\left(x_{1}+x_{2}\right) \in N$ or $b c\left(x_{1}+x_{2}\right) \in N$. If $a c\left(x_{1}+x_{2}\right) \in N$, then $a c x_{2} \notin N$ since $a c x_{1} \notin N$. Since $a R b R c x_{2} \subseteq N$ and $b c x_{2} \notin N$ and $a c x_{2} \notin N$ we have $a b \in\left(N:_{R} M\right)$. Similar to the case $b c\left(x_{1}+x_{2}\right) \in N$, we get $a b \in\left(N:_{R} M\right)$. Hence $\left(N:_{R} M\right)$ is a 2-absorbing ideal of $R$.

Corollary 2.10. [6, Theorem 2.3] If $R$ is a commutative ring and $N$ is a 2absorbing submodule of $M$, then $\left(N:_{R} M\right)$ is a 2-absorbing ideal of $R$.

Remark 2.11. The converse of the above theorem is not true in general.

Proof. Let $p$ be a fixed prime integer. Then $\mathbb{Z}\left(p^{\infty}\right)=\left\{\alpha \in \mathbb{Q} / \mathbb{Z}: \alpha=r / p^{n}+\mathbb{Z}\right.$ for some $r \in \mathbb{Z}$ and $n \geq 0\}$ is a non-zero submodule of $\mathbb{Q} / \mathbb{Z}$. Let $G_{t}=\{\alpha \in \mathbb{Q} / \mathbb{Z}: \alpha=$ $r / p^{t}+\mathbb{Z}$ for some $\left.r \in \mathbb{Z}\right\}$ for all $t \geq 0$. It is well known that each proper submodule of $\mathbb{Z}\left(p^{\infty}\right)$ is equal to $G_{t}$ for some $t \geq 0$. By [6, page 914], $G_{t}$ is not a 2 -absorbing submodule of $\mathbb{Z}\left(p^{\infty}\right)$. We can see that $\left(G_{t}: \mathbb{Z} \mathbb{Z}\left(p^{\infty}\right)\right)=0$ is a 2 -absorbing ideal of $\mathbb{Z}$ for all $t \geq 0$.

Compare the next Proposition with Theorem 2.2 (i) in [6].

Proposition 2.12. The intersection of each pair of prime (completely prime) submodules of an $R$-module $M$ is a 2-absorbing (strong 2-absorbing) submodule of $M$.

Proof. Let $N$ and $K$ be two prime (completely prime) submodules of $M$. If $N=$ $K$, then $N \cap K$ is a prime (completely prime) submodule of $M$ so that $N \cap K$ is 
a 2-absorbing (strong 2-absorbing) submodule of $M$. Assume that $N$ and $K$ are distinct. Since $N$ and $K$ are proper submodules of $M$, it follows that $N \cap K$ is a proper submodule of $M$. Next, let $a, b \in R$ and $m \in M$ be such that $a R b R m \subseteq$ $N \cap K(a b m \in N \cap K)$ but $a m \notin N \cap K$ and $a b M \nsubseteq N \cap K$. Then, we can conclude that

(a): $a m \notin N$ or $a m \notin K$, and

(b): $a b M \nsubseteq N$ or $a b M \nsubseteq K$.

These two conditions give 4 cases:

(1): $a m \notin N$ and $a b M \nsubseteq N$;

(2): $a m \notin N$ and $a b M \nsubseteq K$;

(3): $a m \notin K$ and $a b M \nsubseteq N$;

(4): $a m \notin K$ and $a b M \nsubseteq K$.

Let $N$ and $K$ be two prime submodules. We first consider Case(1). Since $a R b R m \subseteq N \cap K \subseteq N$ and $a m \notin N$, it follows from Proposition 2.5 that $b M \subseteq N$. This is a contradiction because $a b M \nsubseteq N$. Hence Case(1) does not occur. Similarly, Case(4) is not possible.

Next, Case(2) is considered. Again, we obtain that $b M \subseteq N$ and then $b m \in N$. Let $r \in R$. Since $a R b R m \subseteq N \cap K \subseteq K$, it follows that $a R(b r m) \subseteq a R(b R m) \subseteq K$. Hence $a M \subseteq K$ or $b r m \in K$ because $K$ is a prime submodule of $M$. If $a M \subseteq K$, then $a b M \subseteq a M \subseteq K$ contradicts $a b M \nsubseteq K$. Thus brm $\in K$. That is $b R m \subseteq K$. Since $K$ is a prime submodule, $b M \subseteq K$ or $m \in K$. If $b M \subseteq K$, then $a b M \subseteq K$ leading to the same contradiction. Therefore, $m \in K$ and then $b m \in K$. Hence $b m \in N \cap K$. The proof of Case(3) is similar to that of Case(2).

Now, let $N$ and $K$ be completely prime submodules of $M$ and $a b m \in N \cap K \subseteq N$. We consider Case(1): Since $a b m \in N \cap K \subseteq N$ and $N$ completely prime we have $a M \subseteq N$ or $b m \in N$. If $a M \subseteq N$, then $a b M \subseteq a M \subseteq N$ which is not possible. So, suppose $b m \in N$. Now $b M \subseteq N$ or $m \in N$. This is not possible and therefor Case (1) does not occur. Similarly, Case(4) is not possible. Next, Case(2) is considered. We have $a b m \in N \cap K \subseteq K$ and since $K$ is completely prime it follows that $a M \subseteq K$ or $b m \in K$. If $a M \subseteq K$ then $a b M \subseteq a M \subseteq K$ which contradicts $a b M \nsubseteq K$ thus $b m \in K$. From $a b m \in N \cap K \subseteq N$ we have $a M \subseteq N$ or $b m \in N$. Since $a m \notin N$, $a M \subseteq N$ is not possible. Hence $b m \in N \cap K$. The proof of Case(3) is similar to that of Case(2). 
Corollary 2.13. [6, Theorem 2.2 (i)] If $R$ is a commutative ring and $N$ is a prime submodule of $M$ or is an intersection of two prime submodules of $M$, then $N$ is 2-absorbing.

Proposition 2.6 guarantees that every prime (completely prime) submodule is a 2-absorbing (strong 2-absorbing) submodule. The converse does not necessarily hold.

Example 2.14. Consider $\mathbb{Z}_{6}$ as $\mathbb{Z}$-module, $(\overline{0})$ is not a prime (completely prime) submodule of $\mathbb{Z}_{6}$ since $\overline{2} . \overline{3} \in(\overline{0})$ but $\overline{3} \notin(\overline{0})$ and $2 \notin\left((\overline{0})_{\mathbb{Z}}: \mathbb{Z}_{6}\right)=6 \mathbb{Z}$. By Proposition 2.12 $(\overline{0})=(\overline{2}) \cap(\overline{3})$ is a 2-absorbing (strong 2-absorbing) submodule of $\mathbb{Z}_{6}$ as $\mathbb{Z}$-module.

Proposition 2.15. Let $N, W$ be two submodules of an $R$-module $M$ and $N \subseteq W$. If $N$ is a 2-absorbing (strong 2-absorbing) submodule of $M$, then $N$ is a 2-absorbing (strong 2-absorbing) submodule of $W$.

Proof. If $W=M$, then there is nothing to prove. Let $a R b R x \subseteq N(a b x \in N)$, where $a, b \in R, x \in W . N$ is a 2-absorbing (strong 2-absorbing) submodule of $M$, so either $a x \in N$ or $b x \in N$ or $a b \in\left(N:_{R} M\right)$. Since $N \subseteq W$ implies $\left(N:_{R} M\right) \subseteq\left(N:_{R} W\right)$, then either $a x \in N$ or $b x \in N$ or $a b \in\left(N:_{R} W\right)$. Hence $N$ is 2-absorbing (strong 2-absorbing) in $W$.

Lemma 2.16. Let $N$ be a proper submodule of an R-module $M$. Then $N$ is a

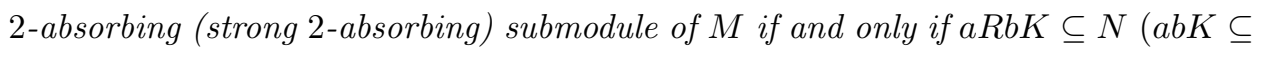
$N)$ implies $a b \in(N: M)$, or $a K \subseteq N$ or $b K \subseteq N$ for each $a, b \in R$ and submodule $K$ of $M$.

Proof. Suppose that $a b \notin(N: M)$ and $a K \nsubseteq N$ and $b K \nsubseteq N$. Then there exist $m_{1}, m_{2}$ in $K$ such that $a m_{1} \notin N$ and $b m_{2} \notin N$. Since $a R b R m_{1} \subseteq a R b K \subseteq N$ $\left(a b m_{1} \in a b K \subseteq N\right)$ and $a b \notin(N: M), a m_{1} \notin N$, we get $b m_{1} \in N$. Also, since $a R b R m_{2} \subseteq a R b K \subseteq N\left(a b m_{2} \in a b K \subseteq N\right)$ and $a b \notin(N: M), b m_{2} \notin N$, we get $a m_{2} \in N$. Now, since $a R b R\left(m_{1}+m_{2}\right) \subseteq a R b K \subseteq N\left(a b\left(m_{1}+m_{2}\right) \in a b K \subseteq N\right)$ and $a b \notin(N: M)$ we have $a\left(m_{1}+m_{2}\right) \in N$ or $b\left(m_{1}+m_{2}\right) \in N$. If $a\left(m_{1}+m_{2}\right) \in N$, i.e. $\left(a m_{1}+a m_{2}\right) \in N$ then since $a m_{2} \in N$ we get $a m_{1} \in N$ which is contradiction. If $b\left(m_{1}+m_{2}\right) \in N$, i.e. $\left(b m_{1}+b m_{2}\right) \in N$, then since $b m_{1} \in N$ we get $b m_{2} \in N$ which is a contradiction. Thus either $a b \in(N: M)$ or $a K \subseteq N$ or $b K \subseteq N$. The converse is clear.

As for the commutative case [6, Theorem 2.4] we have. 
Proposition 2.17. If $N$ is a 2-absorbing submodule of the $R$-module $M$ then $(N$ : $R m)$ is a 2-absorbing ideal of $R$ for every $m \in M \backslash N$.

Proof. Let $a, b, c \in R$ and $m \in M \backslash N$ such that $a R b R c \subseteq(N: R m)$. Hence $a R b(R c R) m \subseteq N$. Since $R c R$ is an ideal of $R$, we have $(R c R) m$ is a submodule of $M$. It now follows from Lemma 2.16 that $a(R c R) m \subseteq N$ or $b(R c R) m \subseteq N$ or $a b M \subseteq N$. Hence $a c R m \subseteq N$ or $b c R m \subseteq N$ or $a b M \subseteq N$. Thus $a c \in(N: R m)$ or $b c \in(N: R m)$ or $a b R m \subseteq a b M \subseteq N$ i.e. $a b \in(N: R m)$ and we are done.

Compare the next Theorem with [7, Theorem 2.3].

Theorem 2.18. Let $N$ be a proper submodule of the $R$ module $M$. If $N$ is 2absorbing (strong 2-absorbing) submodule of $M$ and if $I$ and $J$ are ideals of $R$ and $K$ a submodule of $M$ such that $I J K \subseteq N$, then $I K \subseteq N$ or $J K \subseteq N$ or $I J \subseteq\left(N:_{R} M\right)$. The converse holds for 2-absorbing submodules.

Proof. Suppose $I J K \subseteq N$ and $I J \nsubseteq\left(N:_{R} M\right)$. We show that $I K \subseteq N$ or $J K \subseteq N$. Suppose $I K \nsubseteq N$ and $J K \nsubseteq N$. There exists $a_{1} \in I$ and $a_{2} \in J$ such that $a_{1} K \nsubseteq N$ and $a_{2} K \nsubseteq N$. But $a_{1} R a_{2} K \subseteq I J K \subseteq N\left(a_{1} a_{2} K \subseteq I J K \subseteq N\right)$. Since $N$ is a 2-absorbing (strong 2-absorbing) submodule of $M$ it follows from Lemma 2.16 that $a_{1} a_{2} \in\left(N:_{R} M\right)$. Since $I J \nsubseteq\left(N:_{R} M\right)$. there exists $b_{1} \in I$ and $b_{2} \in J$ such that $b_{1} b_{2} M \nsubseteq N$. Now, since $N$ is 2-absorbing (strong 2-absorbing) and $b_{1} R b_{2} K \subseteq I J K \subseteq N\left(b_{1} b_{2} K \subseteq I J K \subseteq N\right)$ and also $b_{1} b_{2} M \nsubseteq N$ it follows from Lemma 2.16 that $b_{1} K \subseteq N$ or $b_{2} K \subseteq N$. We have the following cases:

Case (1) $b_{1} K \subseteq N$ and $b_{2} K \nsubseteq N$

Since $a_{1} R b_{2} K \subseteq I J K \subseteq N\left(a_{1} b_{2} K \subseteq I J K \subseteq N\right)$ and $a_{1} K \nsubseteq N$ and $b_{2} K \nsubseteq N$ it follows from Lemma 2.16 that $a_{1} b_{2} \in\left(N:_{R} M\right)$. Since $b_{1} K \subseteq N$ and $a_{1} K \nsubseteq N$, we conclude $\left(a_{1}+b_{1}\right) K \nsubseteq N$. On the other hand, $\left(a_{1}+b_{1}\right) R b_{2} K \subseteq N\left(\left(a_{1}+b_{1}\right) b_{2} K \subseteq\right.$ $N)$ and neither $\left(a_{1}+b_{1}\right) K \subseteq N$ nor $b_{2} K \subseteq N$, we get that $\left(a_{1}+b_{1}\right) b_{2} \in(N: M)$ by Lemma 2.16. But since $\left(a_{1}+b_{1}\right) b_{2}=\left(a_{1} b_{2}+b_{1} b_{2}\right) \in(N: M)$ and $a_{1} b_{2} \in(N: M)$, we get $b_{1} b_{2} \in(N: M)$ which is a contradiction.

Case (2) $b_{2} K \subseteq N$ and $b_{1} K \nsubseteq N$

By a similar argument to case (1) we get a contradiction.

Case (3) $b_{1} K \subseteq N$ and $b_{2} K \subseteq N$

$b_{2} K \subseteq N$ and $a_{2} K \nsubseteq N$ gives $\left(a_{2}+b_{2}\right) K \nsubseteq N$. But $a_{1} R\left(a_{2}+b_{2}\right) K \subseteq N$ $\left(a_{1}\left(a_{2}+b_{2}\right) K \subseteq N\right)$ and neither $a_{1} K \subseteq N$ nor $\left(a_{2}+b_{2}\right) K \subseteq N$, hence $a_{1}\left(a_{2}+b_{2}\right) \in$ $(N: M)$ by Lemma 2.16. Since $a_{1} a_{2} \in(N: M)$ and $\left(a_{1} a_{2}+a_{1} b_{2}\right) \in(N: M)$, we have $a_{1} b_{2} \in(N: M)$. Since $\left(a_{1}+b_{1}\right) R a_{2} K \subseteq N\left(\left(a_{1}+b_{1}\right) a_{2} K \subseteq N\right)$ and neither $a_{2} K \subseteq N$ nor $\left(a_{1}+b_{1}\right) K \subseteq N$, we conclude $\left(a_{1}+b_{1}\right) a_{2} \in(N: M)$ by 
Lemma 2.16. But $\left(a_{1}+b_{1}\right) a_{2}=a_{1} a_{2}+b_{1} a_{2}$, so $\left(a_{1} a_{2}+b_{1} a_{2}\right) \in(N: M)$ and since $a_{1} a_{2} \in(N: M)$, we get $b_{1} a_{2} \in(N: M)$. Now, since $\left(a_{1}+b_{1}\right) R\left(a_{2}+b_{2}\right) K \subseteq N$ $\left(\left(a_{1}+b_{1}\right)\left(a_{2}+b_{2}\right) K \subseteq N\right)$ and neither $\left(a_{1}+b_{1}\right) K \subseteq N$ nor $\left(a_{2}+b_{2}\right) K \subseteq N$, we have $\left(a_{1}+b_{1}\right)\left(a_{2}+b_{2}\right)=\left(a_{1} a_{2}+a_{1} b_{2}+b_{1} a_{2}+b_{1} b_{2}\right) \in(N: M)$ by Lemma 2.16. But $a_{1} a_{2}, a_{1} b_{2}, b_{1} a_{2} \in(N: M)$, so $b_{1} b_{2} \in(N: M)$ which is a contradiction. Consequently $I K \subseteq N$ or $J K \subseteq N$.

The converse is clear for the 2-absorbing case since if aRbRm $\subseteq N$, then $(R a R)(R b R) R m \subseteq N$ and we have $a m \in(R a R) R m \subseteq N$ or $b m \in(R b R) R m \subseteq N$ or $a b M \subseteq(R a R)(R b R) M \subseteq N$.

Corollary 2.19. Let $I$ and $J$ be two ideals of $R$ and $P$ a 2-absorbing submodule of $M$. If $m \in M$ such that $I J m \subseteq P$, then $I m \subseteq P$ or $J m \subseteq P$ or $I J \subseteq\left(N:_{R} M\right)$.

Proof. If $I J m \subseteq P$, then $I J R m \subseteq P$ and consequently $I m \subseteq I R m \subseteq P$ or $J m \subseteq J R m \subseteq P$ or $I J \subseteq\left(P:_{R} M\right)$.

Lemma 2.20. Let $I$ be an ideal of $R$ and $N$ be a 2-absorbing (strong 2-absorbing) submodule of $M$. If $a \in R, m \in M$ and IRaRm $\subseteq N(\operatorname{Iam} \subseteq N)$, then am $\in N$ or $\operatorname{Im} \subseteq N$ or $I a \subseteq\left(N_{R}: M\right)$.

Proof. Let $a m \notin N$ and $I a \nsubseteq\left(N_{R}: M\right)$. Then there exists $b \in I$ such that $b a \notin\left(N_{R}: M\right)$. Now, bRaRm $\subseteq N($ bam $\in N)$, implies that $b m \in N$, since $N$ is a 2-absorbing (strong 2-absorbing) submodule of $M$. We have to show that $I m \subseteq N$. Let $c$ be an arbitrary element of $I$. Thus $(b+c) R a R m \subseteq I R a R m \subseteq N$ $(b+c) a m \in \operatorname{Iam} \subseteq N)$. Hence, either $(b+c) m \in N$ or $(b+c) a \in\left(N_{R}: M\right)$. If $(b+c) m \in N$, then by $b m \in N$ it follows that $c m \in N$. If $(b+c) a \in\left(N_{R}: M\right)$, then $c a \notin\left(N_{R}: M\right)$, but $c R a R m \subseteq N($ cam $\in N)$. Thus $c m \in N$. Hence, we conclude that $\operatorname{Im} \subseteq N$.

Corollary 2.21. Let $N$ be a 2-absorbing (strong 2-absorbing) submodule of the $R$-module $M$. Then $(N: M I)=\{m \in M: I m \subseteq N\}$ is a 2-absorbing (strong 2absorbing) submodule of $M$ for every ideal $I$ of $R$.

Proof. Let $I$ be an ideal of $R$ and $a, b \in R$ and $m \in M$ such that $a R b R m \subseteq$ $\left(N:_{M} I\right)\left(a b m \subseteq\left(N:_{M} I\right)\right)$. Thus IaRbRm $\subseteq N(\operatorname{Iabm} \subseteq N)$, then $($ IaR $) R b R m \subseteq$ $I a R b R m \subseteq N$. Hence from Lemma 2.20 we have $(I a R) m \subseteq N$ or $(I a R b) \subseteq\left(N_{R}\right.$ : $M)$ or $b m \in N$. If $b m \in N$, then $I b m \subseteq N$ and consequently $b m \in\left(N:_{M} I\right)$ and we are done. If $I a R b \subseteq\left(N_{R}: M\right)$, then $a b \in a R b \subseteq\left(\left(N:_{R} M\right):_{R} I\right)=\left(\left(N:_{M}\right.\right.$ $\left.I):_{R} M\right)$. If $(I a R) m \subseteq N$, then $a m \in a R m \subseteq\left(N:_{M} I\right)$. Thus $b m \in\left(N:_{M} I\right)$ 
or $a m \in\left(N:_{M} I\right)$ or $a b \in\left(\left(N:_{M} I\right):_{R} M\right)$ which complete the proof for 2absorbing. For Iabm $\subseteq N$ and $N$ strong 2-absorbing it follows from Lemma 2.20 that $a b m \in N$ or $I m \subseteq N$ or $I a b \subseteq\left(N_{R}: M\right)$. If $a b m \in N$, then $a m \in N$ or $b m \in N$ or $a b \in\left(N:_{R} M\right)$. Hence for $a m \in N$ it follows that $I a m \subseteq I N \subseteq N$ and we have $a m \in\left(N:_{M} I\right)$. For $b m \in N$ it follows that $I b m \subseteq I N \subseteq N$ and we have $b m \in\left(N:_{M} I\right)$. For $a b \in\left(N:_{R} M\right)$, we have $a b \in\left(\left(N:_{R} M\right):_{R} I\right)=\left(\left(N:_{M}\right.\right.$ $\left.I):_{R} M\right)$. For $I m \subseteq N$, we have $m \in\left(N:_{M} I\right)$ and thus am $\in\left(N:_{M} I\right)$. For $I a b \subseteq\left(N_{R}: M\right)$, we have $a b \in\left(\left(N:_{R} M\right):_{R} I\right)=\left(\left(N:_{M} I\right):_{R} M\right)$ and $\left(N:_{M} I\right)$ is a strong 2 -absorbing submodule of $M$.

For the next Theorem compare also [7, Theorem 2.7 and Corollary 2.8].

Theorem 2.22. Let $N$ be a 2-absorbing submodule of $M$. Then $\left(N:_{R} M\right)$ is a prime ideal of $R$ if and only if $\left(N:_{R} P\right)$ is a prime ideal for every submodule $P$ of $M$ containing $N$.

Proof. Let $I$ and $J$ be ideals of $R$ and $K$ a submodule of $M$ such that $N \subseteq P$ and $I J \subseteq\left(N:_{R} P\right)$. Hence $I J P \subseteq N$. Since $N$ is a 2-absorbing submodule of $M$ it follows from Theorem 2.18 that $I P \subseteq N$ or $J P \subseteq N$ or $I J \subseteq\left(N:_{R} M\right)$. Hence $I \subseteq\left(N:_{R} P\right)$ or $J \subseteq\left(N:_{R} P\right)$. Also for $I J \subseteq\left(N:_{R} M\right)$ by the assumption that $\left(N:_{R} M\right)$ is a prime ideal, we get $I P \subseteq I M \subseteq N$ or $J P \subseteq J M \subseteq N$. Hence also $I \subseteq\left(N:_{R} P\right)$ or $J \subseteq\left(N:_{R} P\right)$ and we have $\left(N:_{R} P\right)$ is a prime ideal.

This is clear, just take $P=M$.

Proposition 2.23. Let $N$ and $K$ be submodules of an $R$-module $M$ with $K \nsubseteq N$. If $N$ is a 2-absorbing (strong 2-absorbing) submodule of $M$, then $K \cap N$ is a 2-absorbing (strong 2-absorbing) submodule of $K$.

Proof. Since $N$ and $K$ are submodules of $M$ and $K \nsubseteq N, K \cap N$ is a proper submodule of $K$. Assume that $N$ is a 2-absorbing (strong 2-absorbing) submodule of $M$. Let $a, b \in R$ and $x \in K$ be such that $a R b R x \subseteq N(a b x \in N)$. Since $K$ is a submodule of $M, a b K \subseteq K$ and $a x ; b x \in K$. Moreover, since $a R b R x \subseteq K \cap N \subseteq N$ ( $a b x \in K \cap N \subseteq N$ ) and $N$ is a 2-absorbing (strong 2-absorbing ) submodule of $M$, $a b M \subseteq N$ or $a x \in N$ or $b x \in N$. Thus $a b K \subseteq a b K \cap a b M \subseteq K \cap N$ or $a x \in K \cap N$ or $b x \in K \cap N$. Therefore, $K \cap N$ is a 2-absorbing (strong 2-absorbing ) submodule of $K$.

Proposition 2.24. Let $N$ and $K$ be submodules of an $R$-module $M$ with $K \subseteq N$. Then $N$ is a 2-absorbing (strong 2-absorbing) submodule of $M$ if and only if $N / K$ is a 2-absorbing (strong 2-absorbing) submodule of $M / K$. 
Proof. First, assume that $N$ is a 2-absorbing (strong 2-absorbing) submodule of $M$. Then $N / K$ is a proper submodule of $M / K$. Let $a, b \in R$ and $m \in M$ be such that $a R b R(m+K) \subseteq N / K(a b(m+K) \subseteq N / K)$. Let $s ; t \in R$. Thus asbtm $+K=\operatorname{asbt}(m+K) \subseteq a R b R(m+K) \subseteq N / K$. Then there exists $n \in N$ such that asbtm $+K=n+K$ so that $-n+$ asbtm $\in K \subseteq N$ and then asbtm $\in N$. This shows that $a R b R m \subseteq N$. (For the strong 2-absorbing case, abm $+K=$ $a b(m+K) \subseteq N / K$ and then there exists $n \in N$ such that $a b m+K=n+K$ so that $-n+a b m \in K \subseteq N$ and then $a b m \in N)$. As a result, $a m \in N$ or $b m \in N$ or $a b M \subseteq N$ because $N$ is a 2-absorbing (strong 2-absorbing) submodule of $M$. Therefore, $a(m+K) \in N / K$ or $b(m+K) \in N / K$ or $a b(M / K) \subseteq N / K$. Hence $N / K$ is a 2-absorbing (strong 2-absorbing) submodule of $M / K$.

Conversely, assume that $N / K$ is a 2-absorbing (strong 2-absorbing) submodule of $M / K$. Then $N$ is a proper submodule of $M$. Let $a, b \in R$ and $m \in M$ be such that $a R b R m \subseteq N(a b m \in N)$. Then $a R b R(m+K) \subseteq N / K(a b(m+K) \subseteq N / K)$. Since $N / K$ is a 2 -absorbing (strong 2-absorbing) submodule of $M / K$, we obtain that $a(m+K) \in N / K$ or $b(m+K) \in N / K$ or $a b(M / K) \subseteq N / K$. That is $a m \in N$ or $b m \in N$ or $a b M \subseteq N$. This implies that $N$ is a 2-absorbing (strong 2-absorbing) submodule of $M$.

Consider $R=R_{1} \times R_{2}$ where each $R_{i}$ is an associative ring with identity, $M_{i}$ be an $R_{i}$-module where $i=1,2$, and $M=M_{1} \times M_{2}$ be the $R$-module with $\left(r_{1}, r_{2}\right)\left(m_{1}, m_{2}\right)=\left(r_{1} m_{1}, r_{2} m_{2}\right)$ where $r_{i} \in R_{i}, m_{i} \in M_{i}, i=1,2$.

Theorem 2.25. Let $M_{1}$ be an $R_{1}$-module, $M_{2}$ be an $R_{2}$-module, $R=R_{1} \times R_{2}$ and $M=M_{1} \times M_{2}$. Then

(1) $N_{1}$ is a 2-absorbing $R_{1}$-submodule of $M_{1}$ if and only if $N_{1} \times M_{2}$ is a 2absorbing submodule of $M$; and

(2) $N_{2}$ is a 2-absorbing $R_{2}$-submodule of $M_{2}$ if and only if $M_{1} \times N_{2}$ is a 2absorbing submodule of $M$.

Proof. It suffices to prove only part 1. First, assume that $N_{1}$ is a 2-absorbing $R_{1}$-submodule of $M_{1}$. Suppose that $(a, b) R(c, d) R\left(m_{1}, m_{2}\right) \subseteq N_{1} \times M_{2}$ where $(a, b),(c, d) \in R$ and $\left(m_{1}, m_{2}\right) \in M$.

Then $\left(a R_{1} c R_{1} m_{1}, b R_{2} d R_{2} m_{2}\right)=(a, b) R(c, d) R\left(m_{1}, m_{2}\right) \subseteq N_{1} \times M_{2}$, i.e., $a R_{1} c R_{1} m_{1} \subseteq N_{1}$ and $b R_{2} d R_{2} m_{2} \subseteq M_{2}$. Since $N_{1}$ is a 2-absorbing $R_{1}$-submodule of $M_{1}, a c M_{1} \subseteq N_{1}$ or $a m_{1} \in N_{1}$ or $c m_{1} \in N_{1}$. That is $(a, b)(c, d) M=\left(a c M_{1}, b d M_{2}\right) \subseteq$ $N_{1} \times M_{2}$ or $(a, b)\left(m_{1}, m_{2}\right)=\left(a m_{1}, b m_{2}\right) \in N_{1} \times M_{2}$ or $(c, d)\left(m_{1}, m_{2}\right)=\left(c m_{1}, d m_{2}\right) \in$ $N_{1} \times M_{2}$. Therefore, $N_{1} \times M_{2}$ is a 2-absorbing submodule of $M$. Conversely, assume 
that $N_{1} \times M_{2}$ is a 2-absorbing $R$-submodule of $M$. Let $a, b \in R_{1}$ and $m_{1} \in M_{1}$. Assume that $a R_{1} b R_{1} m_{1} \subseteq N_{1}$. Let $x, y \in R_{2}$ and $m_{2} \in M_{2}$. Then $(a, x) R(b, y) R\left(m_{1}, m_{2}\right)=\left(a R_{1} b R_{1} m_{1}, x R_{2} y R_{2} m_{2}\right) \subseteq N_{1} \times M_{2}$. Since $N_{1} \times M_{2}$ is a 2 absorbing $R$-submodule of $M,(a, x)(b, y) M \subseteq N_{1} \times M_{2}$ or $(a, x)\left(m_{1}, m_{2}\right) \in N_{1} \times M_{2}$ or $(b, y)\left(m_{1}, m_{2}\right) \in N_{1} \times M_{2}$. Then $\left(a b M_{1}, x y M_{2}\right)=(a, x)(b, y) M \subseteq N_{1} \times M_{2}$ or $\left(a m_{1}, x m_{2}\right)=(a, x)\left(m_{1}, m_{2}\right) \in$ $N_{1} \times M_{2}$ or $\left(b m_{1}, y m_{2}\right)=(b, y)\left(m_{1}, m_{2}\right) \in N_{1} \times M_{2}$, i.e., $a b M_{1} \subseteq N_{1}$ or $a m_{1} \in N_{1}$ or $b m_{1} \in N_{1}$. Therefore, $N_{1}$ is a 2-absorbing $R_{1}$-submodule of $M_{1}$.

Theorem 2.26. Let $M_{1}$ be an $R_{1}$-module, $M_{2}$ be an $R_{2}$-module, $R=R_{1} \times R_{2}$ and $M=M_{1} \times M_{2}$. Then

(1) $N_{1}$ is a strong 2-absorbing $R_{1}$-submodule of $M_{1}$ if and only if $N_{1} \times M_{2}$ is a strong 2-absorbing submodule of $M$; and

(2) $N_{2}$ is a strong 2-absorbing $R_{2}$-submodule of $M_{2}$ if and only if $M_{1} \times N_{2}$ is a strong 2-absorbing submodule of $M$.

Proof. It suffices to prove only part 1. First, assume that $N_{1}$ is a strong 2absorbing $R_{1}$-submodule of $M_{1}$. Suppose that $(a, b)(c, d)\left(m_{1}, m_{2}\right) \in N_{1} \times M_{2}$ where $(a, b),(c, d) \in R$ and $\left(m_{1}, m_{2}\right) \in M$.

Then $\left(a c m_{1}, b d m_{2}\right)=(a, b)(c, d)\left(m_{1}, m_{2}\right) \in N_{1} \times M_{2}$, i.e., $a c m_{1} \in N_{1}$ and $b d m_{2}$ $\in M_{2}$. Since $N_{1}$ is a strong 2-absorbing $R_{1}$-submodule of $M_{1}$, it follows that $a c M_{1} \subseteq$ $N_{1}$ or $a m_{1} N_{1}$ or $c m_{1} \in N_{1}$. That is $(a, b)(c, d) M=\left(a c M_{1}, b d M_{2}\right) \subseteq N_{1} \times M_{2}$ or $(a, b)\left(m_{1}, m_{2}\right)=\left(a m_{1}, b m_{2}\right) \in N_{1} \times M_{2}$ or $(c, d)\left(m_{1}, m_{2}\right)=\left(c m_{1}, d m_{2}\right) \in N_{1} \times M_{2}$. Therefore, $N_{1} \times M_{2}$ is a 2-absorbing submodule of $M$. Conversely, assume that $N_{1} \times M_{2}$ is a strong 2-absorbing $R$-submodule of $M$. Let $a, b \in R_{1}$ and $m_{1} \in M_{1}$. Assume that $a b m_{1} \in N_{1}$. Let $x, y \in R_{2}$ and $m_{2} \in M_{2}$. Then $(a, x)(b, y)\left(m_{1}, m_{2}\right)=$ $\left(a b m_{1}, x y m_{2}\right) \in N_{1} \times M_{2}$. Since $N_{1} \times M_{2}$ is a strong 2-absorbing $R$-submodule of $M$, it follows that $(a, x)(b, y) M \subseteq N_{1} \times M_{2}$ or $(a, x)\left(m_{1}, m_{2}\right) \in N_{1} \times M_{2}$ or $(b, y)\left(m_{1}, m_{2}\right) \in N_{1} \times M_{2}$. Then $\left(a b M_{1}, x y M_{2}\right)=(a, x)(b, y) M \subseteq N_{1} \times M_{2}$ or $\left(a m_{1}, x m_{2}\right)=(a, x)\left(m_{1}, m_{2}\right) \in N_{1} \times M_{2}$ or $\left(b m_{1}, y m_{2}\right)=(b, y)\left(m_{1}, m_{2}\right) \in N_{1} \times M_{2}$, i.e., $a b M_{1} \subseteq N_{1}$ or $a m_{1} \in N_{1}$ or $b m_{1} \in N_{1}$. Therefore, $N_{1}$ is a 2-absorbing $R_{1}$ submodule of $M_{1}$.

Acknowledgement. The authors would like to thank the referee for the valuable suggestions and comments. 


\section{References}

[1] A. Badawi, On 2-absorbing ideals of commutative rings, Bull. Austral. Math. Soc., 75(3) (2007), 417-429.

[2] A. M. Buhphang and M. B. Rege, Semi-commutative modules and Armendariz modules, Arab J. Math. Sci., 8(1) (2002), 53-65.

[3] A. Y. Darani and F. Soheilnia, 2-Absorbing and weakly 2-absorbing submodules, Thai J. Math., 9(3) (2011), 577-584.

[4] J. Dauns, Prime modules, J. Reine Angew. Math., 298 (1978), 156-181.

[5] N. J. Groenewald and D. Ssevviiri, Completely prime submodules, Int. Electron. J. Algebra, 13 (2013), 1-14.

[6] Sh. Payrovi and S. Babaei, On 2-absorbing submodules, Algebra Colloq., 19 (2012) 913-920.

[7] Sh. Payrovi and S. Babaei, On the 2-absorbing submodules, Iran. J. Math. Sci. Inform., 10(1) (2015), 131-137.

N. J. Groenewald (Corresponding Author)

Department of Mathematics

Faculty of Science

Nelson Mandela University

Port Elizabetyh

South Africa

e-mail: nico.groenewald@mandela.ac.za

Bac T. Nguyen

Nguyen Tat Thanh University

300 A Nguyen Tat Thanh street

Ho Chi Minh city, Vietnam

and Dept. of Basic Science

Thainguyen Univ. of Economics and Business Administration

Thainguyen

Vietnam

e-mail: bacnt2008@gmail.com 